The Astrophysical Journal Supplement Series, 90:837-839, 1994 February

(c) 1994. The American Astronomical Society. All rights reserved. Printed in U.S.A.

\title{
DISK-ACCRETING MAGNETIC NEUTRON STARS AS HIGH-ENERGY PARTICLE ACCELERATORS
}

\author{
Russell J. Hamilton, Frederick K. Lamb, ${ }^{1}$ and M. Coleman Miller \\ Department of Physics, University of Illinois at Urbana-Champaign, 1110 West Green Street, Urbana, IL 61801-3080 \\ Received 1993 February 26; accepted 1993 May 19
}

\begin{abstract}
Interaction of an accretion disk with the magnetic field of a neutron star produces large electromotive forces, which drive large conduction currents in the disk-magnetosphere-star circuit. Here we argue that such large conduction currents will cause microscopic and macroscopic instabilities in the magnetosphere. If the minimum plasma density in the magnetosphere is relatively low $\left(\$ 10^{9} \mathrm{~cm}^{-3}\right)$, current-driven micro-instabilities may cause relativistic double layers to form, producing voltage differences in excess of $10^{12} \mathrm{~V}$ and accelerating charged particles to very high energies. If instead the plasma density is higher $\left(210^{9} \mathrm{~cm}^{-3}\right)$, twisting of the stellar magnetic field is likely to cause magnetic field reconnection. This reconnection will be relativistic, accelerating plasma in the magnetosphere to relativistic speeds and a small fraction of particles to very high energies. Interaction of these high-energy particles with X-rays, $\gamma$-rays, and accreting plasma may produce detectable high-energy radiation.

Subject headings: acceleration of particles - accretion, accretion disks - gamma rays: theory — plasmas radiation mechanisms: nonthermal - stars; neutron
\end{abstract}

\section{INTRODUCTION}

Observations of MeV-GeV gamma rays using the Compton Observatory and GRANAT as well as steady improvement in the sensitivities of ground-based high-energy ( $\mathrm{TeV}-\mathrm{PeV})$ detectors have stimulated interest in accreting neutron star systems as possible sources of detectable fluxes of high-energy electromagnetic radiation. Lamb, Hamilton, \& Miller (1993; see also Miller, Lamb, \& Hamilton 1993b) show that interaction of the magnetic field of a neutron star with the plasma in a Keplerian accretion disk generates an electromotive force $10^{15} \mathrm{~V}$, even if the star is slowly rotating. They argue that the number density of particles in the magnetosphere of a diskaccreting neutron star is likely to be many orders of magnitude larger than the Goldreich-Julian charge density. Some mechanisms advanced to explain particle acceleration in the magnetospheres of isolated rotation-powered pulsars, such as those requiring a charge-separated magnetosphere, are inconsistent with these conditions. We show that charged particles can be accelerated to high energies in the magnetospheres of diskaccreting neutron stars even if the plasma density there is large. Because the resistivity of the disk-magnetosphere-star circuit is small, the electromotive force drives large conduction currents leading to magnetospheric instabilities, such as relativistic double layers and magnetic reconnection events. Acceleration of particles to high energies can result from either of these instabilities. We show that the density of plasma in the magnetosphere determines which instability limits the current. These results have also been described by Hamilton, Lamb, \& Miller (1993a).

\section{MODEL}

Consider a binary system in which a magnetic neutron star is accreting plasma from a geometrically thin Keplerian disk situated in the equatorial plane of the neutron star with its axis of

' Also Department of Astronomy. rotation aligned with that of the star. We analyze this system using cylindrical coordinates $(\varpi, \phi, z)$ centered on the star, with the $z$-axis aligned with the stellar rotation axis. We assume the star has mass $M_{s}$, radius $R_{s}$, and rotates with angular velocity $\boldsymbol{\Omega}_{s}$, and that the stellar magnetic field is dipolar, with the dipole moment $\mu$ aligned with the rotation axis. We assume further that the mass flux $\dot{M}$ through the disk far from the star is constant.

The accreting plasma couples to the neutron star via its magnetosphere. Processes occurring within the magnetosphere mediate the transfer of mass, energy, and angular momentum between the accreting plasma and the star. Far from the star, where the magnetic field is unimportant, the accreting plasma orbits at the local Keplerian velocity $\Omega_{\mathrm{K}}(\boldsymbol{\varpi})$. The Keplerian flow ends at the radius $\varpi_{0}$ where the magnetic stress is so large that it extracts the angular momentum of the disk plasma in a radial distance much less than $\varpi$ (Ghosh \& Lamb 1991). The differential rotation between the star and accreting plasma generates an electromotive force (EMF) of magnitude (Lamb et al. 1993)

$$
\mathscr{E}_{g} \sim \varpi_{0}^{2} \Omega_{\mathrm{K}}\left(\varpi_{0}\right) B_{p}\left(\varpi_{0}\right) / c \sim 10^{15} \varpi_{8}^{-5 / 2} \mu_{30}\left(M_{s} / M_{\odot}\right)^{1 / 2} \mathrm{~V}
$$

in the disk-magnetosphere-star circuit.

Although the magnitude of the charge density in the magnetosphere is expected to be comparable to the Goldreich-Julian value $n_{\mathrm{GJ}} \approx\left|\boldsymbol{\Omega}_{s} \cdot \boldsymbol{B}\right| /(2 \pi e c)$, the number density of ions (and electrons) in the magnetosphere is expected to be much larger. As a result, the EMF drives large conduction currents as shown in Figure 1 for a slowly rotating star. The azimuthal field $B_{\phi}$ produced by the conduction currents can be thought of as resulting from the twisting of the poloidal magnetic field $B_{p}$ by the rotational motion of the accreting plasma relative to the star.

In the absence of other processes, the current asymptotically approaches the steady state value $I_{\mathrm{ss}}=\mathscr{E}_{g} / \mathscr{R}$ with an $e$-folding 


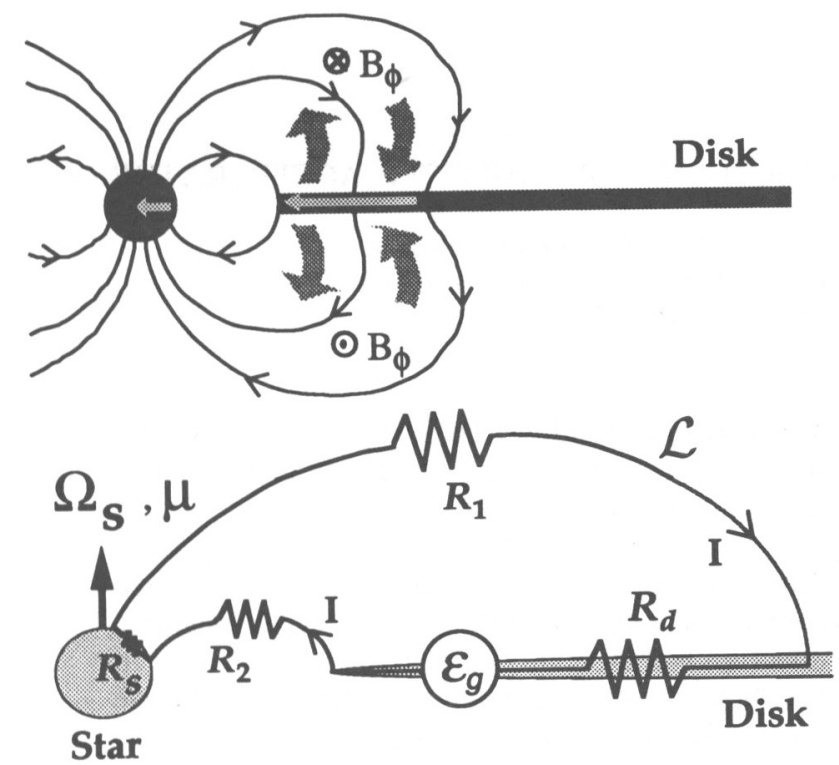

FIG. 1.- (Top) Orbiting motion of the disk plasma generates an EMF that drives cross-field currents (light shaded arrows) within the disk and the neutron star and field-aligned currents (shaded arrows) within the magnetosphere. (Bottom) Equivalent circuit, showing the generated EMF $\mathscr{E}_{g}$, the circuit inductance $\mathscr{L}$, and the resistances $\mathscr{R}_{s}$ and $\mathscr{R}_{d}$ of the star and disk. Also shown are the resistances $\mathscr{R}_{1}$ and $\mathscr{R}_{2}$ and the current $I$ in the two magnetospheric legs of the circuit.

time $\sim \mathscr{L} / \mathscr{R}$, where $\mathscr{R}=\mathscr{R}_{1}+\mathscr{R}_{2}+\mathscr{R}_{d}+\mathscr{R}_{s}$. The total resistance $\mathscr{R}$ in the circuit is very small, so that, in the absence of instabilities, a large asymptotic current $I_{\mathrm{ss}}$ would flow, generating an azimuthal magnetic field $\sim 10^{10}$ times larger than the poloidal field. Because of the low resistance, electrical currents become large enough to produce microscopic and/or magnetohydrodynamic (MHD) instabilities, which can lead to the acceleration of ions and electrons to energies greater than $1 \mathrm{TeV}$.

\section{INSTABILITIES AND PARTICLE ACCELERATION}

We consider the consequences of large electrical currents in the magnetosphere, with emphasis on processes that may lead to particle acceleration. Whether micro-instability or reconnection occurs first depends on the particle density in the most tenuous regions of the magnetosphere. If this particle density is moderately low, field-aligned currents are likely to trigger micro-instabilities and possible double layer formation before reconnection occurs. If instead the lowest particle density is higher, reconnection is likely to occur before the threshold for micro-instability is reached. In some circumstances, the current may continue to grow even after double layers have formed, eventually triggering a reconnection event.

Micro-instabilities will occur if the current density in the magnetosphere is high enough. If the current density exceeds

$$
j_{\text {micro }}=e n_{e}\left(k T_{e} / m_{e}\right)^{1 / 2} \sim 10^{9} n_{9} T_{8}^{1 / 2},
$$

micro-instabilities will be produced. Here $m_{e}, n_{e}=n_{9} 10^{9}$ $\mathrm{cm}^{-3}$, and $T_{e}=T_{8} 10^{8} \mathrm{~K}$ are the electron mass, density, and temperature. These instabilities can produce anomalous resistance, heating, or double layers (see Spicer 1982).

Double layers, which are observed in both space and laboratory plasmas (see Block 1978; Carlqvist 1979), are related to the nonlinear BGK-wave solutions of the Vlasov-Maxwell equations and form when large electrical currents flow in response to applied voltages. These structures consist of two equal but oppositely charged, approximately parallel space charge regions (see Fig. 2). The electron number density $n_{e}$ is many orders of magnitude larger in the disk than in the magnetosphere, while the temperature $T_{e}$ is the same to within a factor of $10^{2}$, so that $j_{\text {micro }}$ is orders of magnitude smaller in the magnetosphere than in the disk. Thus, formation of double layers is most likely to occur in the magnetosphere. In these systems, we expect double layers to be strong (voltage $V_{\mathrm{DL}} \gg$ $\left.k T_{e}\right)$ and relativistic $\left(V_{\mathrm{DL}} \gg m_{p} c^{2} \gg m_{e} c^{2}\right)$. Double layers are therefore effective particle accelerators.

Carlqvist (1969) found that for a relativistic double layer bordered by a thermal plasma consisting of ions and electrons with the ion mass $m_{i} \gg m_{e}$, the voltage drop is related to the current density by

$$
V_{\mathrm{DL}} \approx\left(\pi m_{i} c j d^{2} / e\right)^{1 / 2},
$$

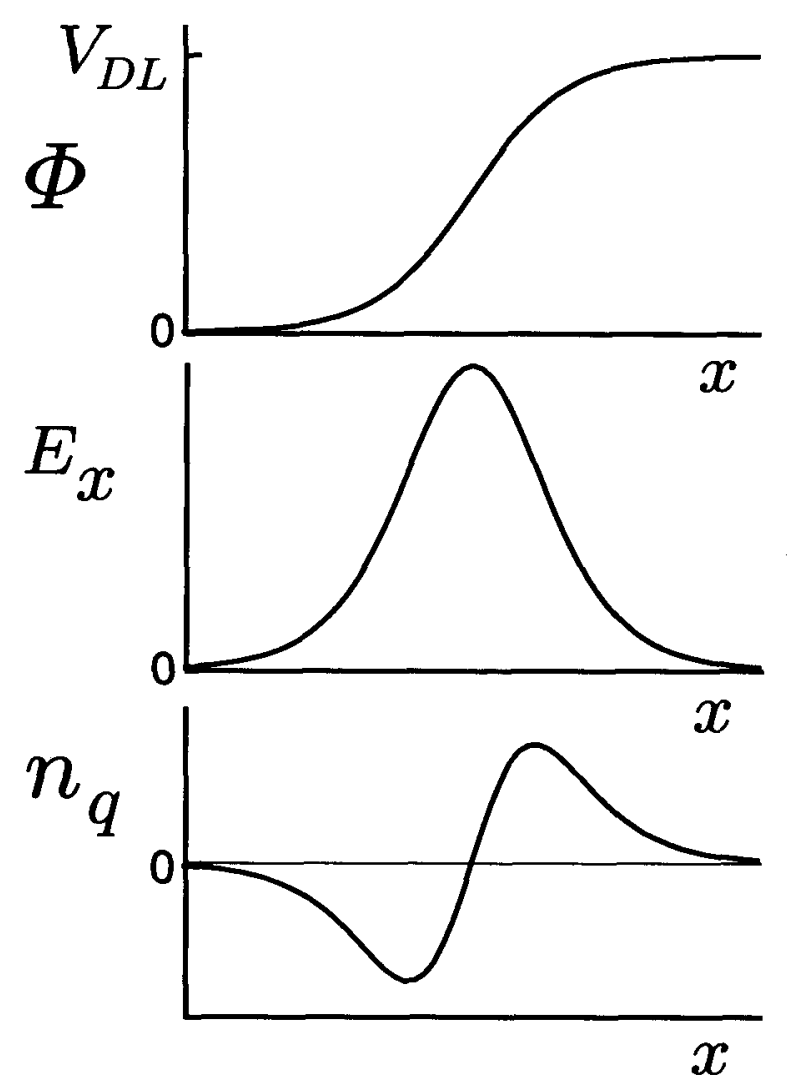

FIG. 2.-Example of a stationary double layer solution, showing the electrical potential $\phi$, normal electric field $E_{x}$, and charge density $n_{q} \equiv n_{+}-$ $n_{-}$as functions of position $x$. Charge separation is maintained by a balance between inertial and electrostatic forces. Quasi-neutrality is violated at both edges of the layer, but the spatially integrated charge is almost zero, so the electric field outside the layer is small. Such a layer produces approximately monoenergetic electrons and protons ( for $e V_{\mathrm{DL}} \gg m_{p} c^{2}$ ), since in moving across the layer both attain energies $\sim e V_{\mathrm{DL}}$. 
where $d=d_{8} 10^{8} \mathrm{~cm}$ is the width of the double layer. For a current density equal to $j_{\text {micro }}$, the voltage drop is $\sim 10^{13} d_{8} n_{9}^{1 / 2} T_{8}^{1 / 4} \mathrm{~V}$.

The circuits linking the neutron star, magnetosphere, and disk are highly inductive. Therefore current disruption, which may occur if the current density greatly exceeds $j_{\text {micro }}$, would cause the energy stored in these circuits $\left(\sim \mathscr{L} I^{2} / 2\right)$ to be released rapidly via a discharge process in the region of the disruption. Such an event has been called an "exploding double layer" by Alfvén (1981) and would produce voltage drops across the double layer $\sim \mathscr{L} d I / d t$. Assuming that the current density is $j_{\text {micro }}$ and that the current changes on a time scale $d / c$, the voltage drop across such an exploding layer is $\sim 10^{15} \mathrm{~V}$.

Another possibility is that the continual twisting of the magnetic field by the differential rotation of the accreting plasma and the star causes the magnetic field configuration to become unstable to reconnection (see Parker 1979), since the field cannot be wound up indefinitely. We expect the magnetic field to become unstable to reconnection when twisting of the poloidal field produces an azimuthal field that exceeds the poloidal field by a factor $\gamma_{\text {mhd }} \sim 10$. The electrical current density corresponding to this value of $\gamma_{\mathrm{mhd}}$ is

$$
j_{\mathrm{mhd}} \sim\left(c \gamma_{\mathrm{mhd}} B_{p}\right) /\left(4 \pi \varpi_{0}\right) \sim 3 \times 10^{9}\left(\gamma_{\mathrm{mhd}} / 10\right) B_{6} \varpi_{7}^{-1} .
$$

If magnetic reconnection occurs steadily, it mainly produces heating of the plasma and bulk acceleration to the Alfvén velocity, $v_{\mathrm{A}}=c\left[B^{2} /\left(4 \pi n_{e} m_{p} c^{2}+B^{2}\right)\right]^{1 / 2}$, with a small fraction of particles accelerated to higher energies. In the magnetosphere, $B^{2} \gg 4 \pi n_{e} m_{p} c^{2}$, so the resulting bulk plasma motion is relativistic, with Lorentz factor $\gamma_{\mathrm{A}} \approx 10^{2} n_{9}^{-1 / 2} B_{6}$. The corresponding electron and proton energies are $\sim 10^{8}$ and $\sim 10^{11} \mathrm{eV}$, respectively. If instead magnetic reconnection occurs in flaring processes, voltages comparable to the full EMF $\left(\sim 10^{15} \mathrm{~V}\right)$ may occur.
In summary, rotation of the disk plasma relative to the star continually twists the magnetic field, steadily increasing the field-aligned currents in the magnetosphere. Because the internal resistance of the disk-star generator is so low, these currents become large enough to trigger processes that accelerate particles. The detailed behavior of the system depends on what mechanism limits the current. Comparison of equations (2) and (5) shows that there are two regimes. If the particle density in the most tenuous regions of the magnetosphere is moderate $\left(n \lesssim 10^{9} \mathrm{~cm}^{-3}\right.$ ), field-aligned currents are likely to trigger micro-instabilities that may produce relativistic double layers. If the particle density is higher $\left(n \gtrsim 10^{9} \mathrm{~cm}^{-3}\right)$, we expect magnetic reconnection to occur first. In either case, ions and electrons will be accelerated.

To determine the energies that particles can attain, their acceleration rates must be compared with their energy loss rates. In the magnetosphere, ions are freely accelerated to the full voltage drop. For electrons, energy losses are important. Emission of curvature radiation limits the electrons to energies $\lesssim 10^{13} \mathrm{eV}$. Emission of synchrotron radiation forces electrons to move along the field, but does not limit their acceleration along the field. Inverse Compton drag does not limit the energy here because electron-photon scattering is in the extreme Klein-Nishina regime. Gamma-rays are produced by interaction of the accelerated ions and electrons with $\mathrm{X}$-rays and accreting plasma. A more detailed discussion of photonelectron interactions important for understanding the observable radiation expected from these accreting neutron stars is given elsewhere (Miller, Lamb, \& Hamilton 1993a; Hamilton, Lamb, \& Miller 1993b).

This research was supported in part by NSF grant PHY 91 00283 and NASA grant NAGW 1583 at the University of Illinois.
Alfvén, H. 1981, Cosmic Plasma (Dordrecht: Reidel)

Block, L. P. 1978, Ap\&SS, 55, 59

Carlqvist, P. 1969, Sol. Phys., 7, 377

. 1979, in Wave Instabilities in Space Plasmas, ed. P. J. Palmadesso \& K. Papadopoulos (Dordrecht: Reidel), 83

Ghosh, P., \& Lamb, F. K. 1991, in Neutron Stars: Theory \& Observation, ed. J. Ventura \& D. Pines (Dordrecht: Kluwer), 363

Hamilton, R. J., Lamb, F. K., \& Miller, M. C. 1993a, in Proc. Compton Observatory Symposium, ed. N. Gehrels (New York: AIP), in press

. $1993 \mathrm{~b}$, in preparation

\section{REFERENCES}

Lamb, F. K., Hamilton, R. J., \& Miller, M. C. 1993, in Isolated Pulsars, ed. K. Van Riper, R. Epstein, \& C. Ho (Cambridge: Cambridge Univ. Press), in press

Miller, M. C., Lamb, F. K., \& Hamilton, R. J. 1993a, in Proc. Compton Observatory Symposium, ed. N. Gehrels (New York: AIP), in press - 1993b, ApJS, 89, 00

Parker, E. N. 1979, Cosmical Magnetic Fields (Oxford: Clarendon)

Spicer, D. S. 1982, Space Sci. Rev., 31, 351 